\title{
INTERINATIONAL FULL-SCALE TEST FACILITY FOR STRUCTURAL CONTROL
}

\author{
James L. Beck and Wilfred D. Iwan \\ Division of Engineering and Applied Science \\ California Institute of Technology \\ Pasadena, California 91125, USA \\ Jay-Chung Chen \\ Research Center and Department of Mechanical Engineering \\ Hong Kong University of Science and Technology \\ Clear Water Bay, Kowloon, Hong Kong
}

\begin{abstract}
Planning is underway to establish an international full-scale test facility for structural control on the campus of the Hong Kong University of Science and Technology. The purpose of the test facility is to facilitate the development and application of structural response control and health monitoring technologies to improve the safety, serviceability and economy of structures required to resist wind loads, as well as the development of wind engineering information under various wind conditions. The test facility will be centered around a 30-meter high (ten-story) structure with state-of-the-art equipment for the experimental study of structural response control and realtime health monitoring, and it will be available for use by the internaticnal structural control community. Current plans are described for the full-scale test structure and associated test facilities, and for a proposed research program, international cooperation and industrial participation.
\end{abstract}

\section{Introduction}

Active control of structural vibrations is a new technology which can make a major impact on the design of civil structures, such as buildings and bridges, to resist severe environmental loads produced by earthquakes and strong winds. Almost all existing structural systems are "passive" in that they withstand loads through their inherent stiffness, damping and strength properties. These features are determined before operation of the structure and remain unchanged (or decrease) throughout the life of the structure. In contrast to this passive design philosophy, new "intelligent" or "smart" structural systems can be considered which can actively alter their stiffness and damping characteristics, and even their configu- ration, to reduce the negative influences of external loads that they sense. This concept of active structural control provides an oppportunity to enhance a structure's resistance to external loads by augmenting conventional passive structural design. In addition to having the potential to enhance the safety and integrity of structures under severe, but rare, loading events, active control of vibrations can also be used to increase the comfort of occupants in tall buildings which tend to be "lively" under wind levels which may occur quite frequently.

Structures can also be made "intelligent" or "smart" in another way, by self-monitoring of their structural "health" or condition. This technology draws on recent advances in digital data acquisition systems, field-worthy high-speed microprocessors, massstorage devices, data transmission capabilities, and on-line system identification techniques, to allow realtime monitoring and automated decision making. For example, rapid automated warnings, and other emergency actions, can be made in response to extreme loading events on a structure, such as typhoons/hurricanes, tornadoes, earthquakes, explosions and impacts. These warnings may provide an alert of the intensity of the structural response to the event, and they may also indicate the presence of damage. In addition, continuous monitoring provides the potential to detect and locate damage due to deterioration from long-term environmental effects, such as material fatigue or corrosion, so that a rapid automated response could be made to avoid loss of life and reduce the economic consequences of the damage.

The potential benefits of active structural control and continuous real-time monitoring of civil structures are so great that active research programs are being carried out in many countries. For example, a U.S. Panel 
on Structural Control Research has been set up with support from the National Science Foundation, and the Panel organized a U.S. National Workshop on Structural Control Research in Los Angeles in October, 1990, which drew over 100 interested participants [1]. The Japanese have also set up a Panel on Structural Response Control supported by the Japan Science Council which is actively promoting this technology. To encourage international interaction and cooperation in these activities, the International Association for Structural Control (IASC) was formed in August, 1993, at an International Workshop on Structural Control in Hawaii [2]. The first organized event of the IASC is the First World Conference on Structural Control in Los Angeles, August 3-5, 1994.

Although there is an increasing interest in "smart structures" technologies, the number of applications in civil structures is still rather small. Examples include active control systems installed in a few buildings in Japan to enhance human comfort by reducing ambient vibrations from wind and mild earthquakes, and their temporary use during the vulnerable construction stages of some bridges in Japan. There are also structural monitoring systems planned for several large bridges in the USA and in Asia.

Structural engineering professionals exhibit an appropriate conservatism to the adoption of new technologies which can affect the safety and integrity of the structures that they design and build. Civil structures typically represent a large investment and place a large number of people at risk if they should fail. To get promising new concepts implemented in real structures, they must be successfully taken through several stages of development: theoretical studies with numerically simulated data, then laboratory tests on reduced scale models, and finally, experiments on full-scale test structures. It is only when the benefits, safety and reliability of a new technology have been successfully demonstrated in this way that the structural engineering profession will start to give it serious consideration. Such evolutionary steps were performed over the last two decades for a passive control technology called "base isolation", which is used to reduce the earthquake attack on buildings by providing a flexible interface between the base of the structure and the ground. Applications of base isolation have become sufficiently widespread that their design is now covered by building codes in several seismically-active countries. In the case of active control technologies, the evolutionary steps are of even greater importance for these technologies to gain acceptance because of their potential to destabilize the structure if they malfunction so as to increase the vibrational energy rather than dissipate it.
To facilitate the application of structural response control and health monitoring technologies to improve the safety, serviceability and economy of civil structures, it is therefore desirable to have dedicated full-scale structures with state-of-the-art equipment which are available for testing and demonstration of the effectiveness of these technologies. Since the construction of such a structure requires a large investment of resources, it is attractive to consider a facility which would be available for use by the international structural control community, not only because this allows funds from many different sources to be pooled, but also because this would ensure that the facility is continuously utilized.

Hong Kong has one of the most active construction industries and one of the greatest concentrations of high-rise buildings in the world. Of its 6 million people, some $90 \%$ live and work on $15 \%$ of its 450 square miles of land. High-rise structures for residential, commercial and industrial purposes make this high population density possible. At the same time, Hong Kong is located in a region of strong winds, buffeted by a northeast monsoon in the winter, with occasional gusts of over 100 knots, and by typhoons from the South China Sea throughout the summer. On average, Hong Kong suffers a direct hit from a typhoon every five years and several near-misses each year. In addition, because of the rapid economic growth that has occurred in Hong Kong, major infrastructure developments are taking place, so there are many opportunities for the implementation of proven new technologies. Hence, Hong Kong offers a natural and man-made environment which is ideal for the support and application of structural response control and monitoring technologies.

In December 1991, just three months after its official opening, the Hong Kong University of Science and Technology hosted an International Workshop on Technology for Hong Kong's Infrastructure Development [3]. One of the recommendations from this workshop was that an international test facility for structural control and health monitoring technologies should be constructed in Hong Kong. In response to this recommendation, the Hong Kong University of Science and Technology (HKUS'T), in cooperation with the California Institute of Technology, organized the Planning Workshop for the Hong Kong International Full-Scale Structural Control Test Facility in December, 1993. The purpose of this workshop was to develop plans for implementing such a facility. At the workshop, nearly 40 international experts in structural control, structural engineering and wind engineering developed preliminary plans for a full-scale test structure and associated test facilities on the campus of HKUST, and for a proposed re- 
search program, international cooperation and industrial participation [4]. In this paper, an overview of these plans is presented.

\section{Purpose and Goals}

The purpose of the Hong Kong International FullScale Structural Control Test Facility is to facilitate the developmert and application of structural response control and health monitoring technologies to improve the safet $y$, serviceability and economy of structures required to resist wind loads, as well as the development of wind engineering information under various wind conditions. It is hoped that this experimental test facility will not only foster the advancement, innovation and application of structural response control and health monitoring technologies, but will also serve as an international focal point which promotes interaction between the educational community, industry: and the public sector.

The primary technical goals are:

- To develop structural response control strategies and device; which can mitigate the effects of environmential loads.

- To evaluate the effectiveness and reliability of active, passive and hybrid control devices (including aerodynamic control) under actual severe environmental conditions.

- To develop and evaluate methods and devices for continuous real-time monitoring and identification of the state and health of structures.

- To improve understanding of local strong wind climatology and the effects of strong winds on structures, and to develop improved techniques for measurement of wind loads on structures.

\section{Location of Test Facility}

The test facility will be constructed on the HKUST campus at Clear Water Bay, near Sai Kung on the eastern shore of the Kowloon peninsula. It is a wellexposed area with prevailing easterly winds which is subjected to strong winds during the annual typhoon season. Also, complementary facilities are available at HKUST, such as a structural dynamics laboratory, a wind tunnel, and extensive computer systems.

\section{Test Structure}

The test structure will be approximately 30 meters (ten stories) tall, witl $1 \times 1$ or $1 \times 2$ bays, each $4-6$ meters wide. The structure will be built with a steel moment-resisting spare frame, and the cladding will be non-porous, replaceable or reconfigurable, and possibly automated to provide active appendages for direct aerodynamic control.
The dynamic properties of the test structure will reflect the dynamic behavior of high-rise buildings. For example, it is expected that there will be at least five significant sway modes in each of two perpendicular directions, plus five significant torsional modes about a vertical axis; with at least three modes of each set having natural frequencies less than $10 \mathrm{~Hz}$. The lowest frequencies associated with each set of modes will be less than $0.7 \mathrm{~Hz}$, and the fundamental frequency of the structure will be no more than $0.5 \mathrm{~Hz}$. The goal will be to design the structure so that its lower mode frequencies are in the most energetic part of the wind power spectrum to the maximum extent possible. Finally, the structure will incorporate removable or replaceable structural elements to allow the natural frequencies and mode shapes to be changed; for example, it should be possible to adjust some of the modes which are dominant in different directions so that they have the same natural frequency.

\section{Instrumentation and Equipment}

Two basic control systems will be available for tests: an active mass damper and an active tendon control system, which will use a set of standard actuators (servomotors and electrohydraulic actuators respectively) that can be moved about in the structure. Actuators will also be available for forced excitation by using either a reference structure as a "reaction wall" or using active mass "shakers". This will facilitate the identification of high-fidelity structural models, as well as the simulation of environmental loads on the structure during initial testing of new systems.

The structure will be equipped with acceleration, velocity and displacement transducers, load cells, and strain gauges, with built-in flexibility to change the sensor locations. Wind instrumentation will include sensors which are capable of measuring the spectral content of the wind speed up to $10 \mathrm{~Hz}$ and which are positioned at several elevations on an upstream meterological tower. There will also be pressure sensors distributed over the structure to measure the aerodynamic forces.

A remote control room will contain a computer system for real-time data acquisition, processing and multiple displays, as well as a computer for on-line control of the actuators. The data acquisition system and sensors will have sufficient dynamic range to measure vibrations and wind excitation from low levels to strong wind gusts, and sufficient bandwidth and resolution to permit accurate determination of the structural response and wind excitation. An installed network of data lines will accommodate several hundred channels and there will be sufficient real-time recording capacity to cover all these channels. Close-circuit 
TV with monitors in the control room may be used during testing in high winds.

The controller will consist of an expandable highspeed digital signal processor which is programmable via a standard simulation language. It will have sufficient resolution, dynamic range and bandwidth to accurately reproduce the desired control law.

\section{Wind Environment at Test Site}

Since wind measurement during a typhoon is difficult and dangerous, not much is currently known about wind characteristics, such as gusts speeds, turbulence, wind direction and vertical profile, at the planned site at HKUST. Rugged equipment on a meterological tower will be installed as soon as possible to begin to measure wind characteristics at heights relevant to the full-scale structure. Fluctuation frequencies less than $10 \mathrm{~Hz}$ will be of principal concern. Wind profiles at higher altitudes will be measured at reduced resolution by remote sensing using radio waves and acoustic sodar.

Wind models developed for typhoons and hurricanes will be compared to field data, and their suitability for site-specific application will be evaluated. The wind model will be adjusted, if necessary, based on the field data gathered at the site during the early stages of the project. This standardized model and its associated database will be available to participating experimenters.

\section{Research Program}

The research program will focus primarily on:

- Active control strategies to reduce vibration response, including studies of such factors as robustness and adaptation, control-structure interaction, model reduction, centralized and decentralized control, performance measures, and optimal sensor and actuator locations

- Mechanisms for generating forces, such as tendons, braces, moving masses, gas-jet pulses, aerodynamic appendages, and variable stiffness mechanisms

- Actuator technology, such as servomotors, electro-hydraulic and electro-rheological devices, and "smart material" devices

- Passive, semi-active and hybrid (active-passive) control technologies to reduce vibration response, such as viscoelastic dampers, tuned mass dampers, variable stiffness and damping devices, and hybrid mass dampers

- System identification and state estimation methods to improve the performance of controllers
- Health-monitoring technology for detection of deterioration, damage and failure of the structural system and control system

- Measurement and analysis technologies to improve understanding of the wind environment and aerodynamic forces on structures

- Measurement and analysis technologies for structural response

- Effectiveness and long-term reliability of control systems for civil structures under severe environmental conditions

- Mechanisms and methods for generating representative environmental loads for testing

A supporting research effort will be devoted to fundamental studies in structural modeling, mathematical analysis methods, control and system identification theory, computational fluid dynamics, wind tunnel investigations, wind engineering, model and simulation studies, and cost effectiveness analyses. These studies will be analytical or numerical, or will use other experimental facilities.

During the first two years of the program, the first priority is to design and construct a structural control test facility which allows the stated goals to be achieved. To this end, various interacting requirements must be considered related to wind loading and similitude, structural engineering and dynamics, and control and sensor technology, while including constraints due to architectural, operational and economic considerations. These requirements must be balanced during the design of the facility, and this process will be treated as an integral part of the total research effort.

At appropriate stages during the first two years, the following initial experiments will be performed:

- Wind measurement using a meterological tower to establish an initial wind environment database

- Wind tunnel investigations to define the optimal configuration and distribution of pressure transducers over the test structure

- "Calibration" experiments to establish models and properties of the sensor and actuator characteristics

- Proof tests of installed actuators, the control system, and the structural protection mechanism using the forced excitation devices

- Structural tests to establish a high-fidelity standardized dynamic structural model by modal identification and updating of a finite-element model, and by direct measurement of frequency response functions. 
- A complete "shake-down" structural control experiment

\section{International Cooperation and Industrial Participation}

The planned test facility will be open to use by researchers and practitioners from around the world. To encourage international participation, HKUST will collaborate with key international organizations and agencies in cooperative research programs. Organizations to be approached will include the International Association of Structural Control, as well as various government agencies, corporations and technical societies in Hong Kong, the United States, Japan, China and other countries, which have interests in structural control or wind engineering research.

An industrial affiliation is planned to encourage worldwide participation by industry and to tap industrial resources. This will also promote technology transfer of the results of the research program at the facility.

\section{Administration and Management}

The facility will be managed by the Institute of Infrastructure Studies at HKUST, and technicians and clerical staff will be assigned to support the operations of the facility. The Director of this Institute will appoint two advisory committees which will include internationally-known contributors to research and practice in structural response control and monitoring, and wind engineering. One committee will advise the Director on management policies for the test facility, and the other will serve as a research program advisory committee to review and prioritize proposals submitted for use of the facility in response to an annual call for proposals.

The first proposals will be requested during the establishment of the test facility. Submitted proposals will be judged on the basis of their compatibility with the stated goals and research priorities, technical content, the probability of successful completion, and the potential for practical implementation. Those experiments which are selected will be expected to undergo a qualification procedure. This may involve the submission of documented simulation studies of the proposed experiment using the standardized dynamic model for the structure, the standardized wind model, and the calibrated sensor and actuator characteristics. These simulation studies would be expected to include a study of time-delay effects as well as simulated noise and model errors considered possible for the test structure and control system. Periodically, an international meeting will be held at HKUST to present the results of projects completed, or underway, at the facility. Other technical papers related to the goals of the facility will be encouraged.

\section{Conclusion}

Construction of the full-scale structural test facility will begin as soon as the detailed design is complete and capital funding is secured. Preliminary estimates of the total cost are approximately HK $\$ 40$ million (US $\$ 5$ million), to cover design and construction, instrumentation and equipment, and the initial experiments. It is anticipated that the capital for construction of the facilities will come primarily from local developers and construction companies, as well as from international companies interested in structural control and monitoring technologies. Support funds for operating expenses and experiments will come from HKUST, grants and contracts, and user fees for the facility which will be charged as appropriate and necessary.

We welcome input from the structural control re search community on these plans and on possible projects utilizing the planned test facility.

\section{References}

[1] G.W. Housner and S.F. Masri (Eds), Proc. U.S. National Workshop on Structural Control Research, University of Southern California, Los Angeles, October, 1990.

[2] G.W. Housner and S.F. Masri (Eds), Proc. International Workshop on Structural Control, Hawaii, University of Southern California, Los Angeles, August, 1993.

[3] J. C. Chen and J.L. Beck (Eds), Proc. International Workshop on Technology for Hong Kong's Infrastructure Development, Hong Kong University of Science and Technology, Hong Kong, December, 1991.

[4] Hong Kong University of Science and Technology, Proc. Planning Workshop for Hong Kong International Full-Scale Structural Control Test Facility, Hong Kong, December, 1993. 